\title{
Targeting Bruton's tyrosine kinase signaling as an emerging therapeutic agent of B-cell malignancies (Review)
}

\author{
BING XIA*, FULIAN QU*, TIAN YUAN and YIZHUO ZHANG \\ Department of Hematology, Tianjin Medical University Cancer Institute and Hospital, \\ National Clinical Research Center of Cancer, Key Laboratory of Cancer Prevention and Therapy, Tianjin 300060, P.R. China
}

Received November 4, 2014; Accepted September 14, 2015

DOI: 10.3892/ol.2015.3802

\begin{abstract}
It is becoming increasingly evident that B-cell receptor (BCR) signaling is central to the development and function of B cells. BCR signaling has emerged as a pivotal pathway and a key driver of numerous B-cell lymphomas. Disruption of BCR signaling can be lethal to malignant B cells. Recently, kinase inhibitors that target BCR signaling have induced notable clinical responses. These inhibitors include spleen tyrosine kinase, mammalian target of rapamycin, phosphoinositide 3'-kinase and Bruton's tyrosine kinase (BTK). Ibrutinib, an oral irreversible BTK inhibitor, has emerged as a promising targeted therapy for patients with B-cell malignancies. The present review discusses the current understanding of BTK-mediated BCR signaling in the biology and pathobiology of normal and malignant B cells, and the cellular interaction with the tumor microenvironment. The data on ibrutinib in the preclinical and clinical settings is also discussed, and perspectives for the future use of ibrutinib are outlined.
\end{abstract}

\section{Contents}

1. Introduction

2. Overview of the BCR signaling pathway and BTK

3. Preclinical data on ibrutinib in the treatment of B-cell malignancies

4. Clinical trials of ibrutinib in the treatment of B-cell malignancies using monotherapy and combination regimens

5. Conclusions

Correspondence to: Professor Yizhuo Zhang, Department of Hematology, Tianjin Medical University Cancer Institute and Hospital, National Clinical Research Center of Cancer, Key Laboratory of Cancer Prevention and Therapy, Huanhu Western Road, Hexi, Tianjin 300060, P.R. China

E-mail: yizhuozhang111@163.com

*Contributed equally

Key words: B-cell malignancies, ibrutinib, signal transducing, tumor microenvironment, treatment

\section{Introduction}

B-cell malignancies are a heterogeneous group of disorders and treatment of these conditions has essentially remained the same for $>30$ years, with the exception of the inclusion of monoclonal anti-cluster of differentiation (CD)20 agents in combination strategies $(1,2)$. A pivotal moment in the development of novel drugs for this group of disorders arose with the introduction of the first biologic targeted agent, the anti-CD20 monoclonal antibody rituximab, with improved outcomes in almost every B-cell disorder in which it was applied $(3,4)$. However, although improved survival was obtained for the B-cell lymphoma patients, the majority continued to relapse following standard chemo-immunotherapy, and currently, $>15,000$ patients still succumb to B-cell malignancies each year in the United States. Over the past few years, marked progress has been made in understanding a number of the key pathways that drive proliferation, survival and resistance in lymphoma and leukemia (5-7). Our understanding of oncogenic mechanisms has recently been greatly accelerated by the advent of functional and structural genomics. The identification and validation of a number of essential pathways that drive malignant B-cell lymphoma progression have allowed medical chemists to create highly specific small-molecule inhibitors $(8,9)$. In addition, the effect that the tumor microenvironment (TME) has on tumor survival, proliferation and therapy resistance is being increasingly understood and appreciated (10).

Among the most widely studied oncogenic pathways in $\mathrm{B}$-cell lymphomas is the $\mathrm{B}$-cell receptor (BCR) signaling pathway, which has emerged as a crucial player in the survival, proliferation and trafficking of malignant B cells $(11,12)$. Inhibitors of the key kinases in the BCR signaling pathway, including spleen tyrosine kinase (SYK), mammalian target of rapamycin (mTOR), phosphoinositide 3'-kinase (PI3K) and Bruton's tyrosine kinase (BTK), have become a focus of notable clinical interest due to their striking clinical responses $(13,14)$. A certain degree of the efficacy of these agents is due to the attenuation of BCR-dependent lymphoma-TME interactions. The present review discusses the pivotal role of BCR signaling in the integration of intrinsic and extrinsic determinants of TME-mediated lymphoma survival and drug resistance, and examines the use of the BTK inhibitor, ibrutinib, as an example of a promising targeted therapy and future treatment strategy. 


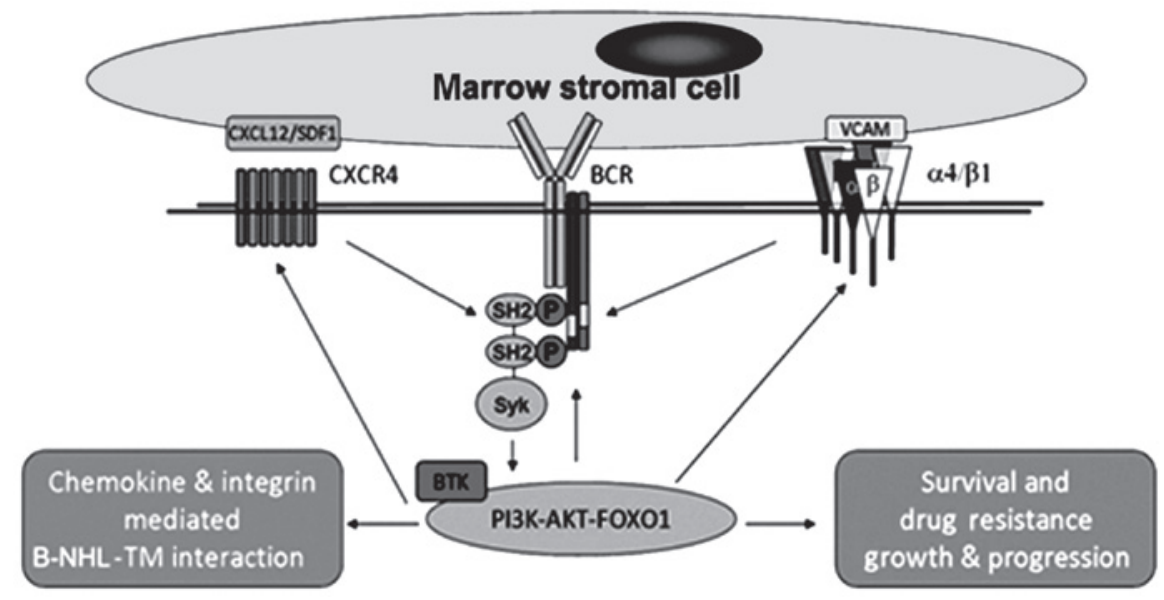

Figure 1. BCR is a central mediator of malignant B-cell homing, survival and microenvironment-mediated drug resistance. BCR, B-cell receptor; SYK, spleen tyrosine kinase; BTK, Bruton's tyrosine kinase; PI3K, phosphoinositide 3'-kinase; SH2, Src Homology 2; CXCR4, chemokine (C-X-C motif) receptor 4; CXCL12/SDF1, C-X-C motif chemokine ligand 12/stromal cell-derived factor 1; VCAM, vascular cell adhesion protein 1; FOXO1, forkhead box protein O1; P, phosphate; B-NHL-TM, B-cell-non-Hodgkin's lymphoma and tumor microenvironment interaction.

\section{Overview of the BCR signaling pathway and BTK}

Normal B cells utilize the BCR pathway to promote the proliferation, differentiation and function of cells, including antibody production $(15,16)$. A simplified version of the BCR pathway and its role in lymphoma cell interactions with TME is illustrated in Fig. 1. Recently, BCR signaling has emerged as a pivotal pathway and is likely to be a key driver of a number of B-cell lymphomas (17). Kinase inhibitors that target BCR signaling have induced striking clinical responses (18). When it is activated, BCR recruits SYK kinase from the cytoplasm to the perimembrane location to become BCR signalosome (19). SRC then phosphorylates SYK, and is auto-phosphorylated and activated prior to interacting with and catalyzing the phosphorylation of several other signaling molecules, including PLC $\gamma 2$ (a lipase), BTK and B-cell linker protein (an adaptor molecule) (20-22). Phosphatidylinositol 4,5-bisphosphate is cleaved into diacylglycerol and inositol triphosphate by activated PLC $\gamma 2$, which results in the mobilization of calcium and the activation of a number of downstream signaling pathways, such as the AKT, mitogen-activated protein kinase (MAPK) and nuclear factor- $\kappa \mathrm{B}(\mathrm{NF}-\kappa \mathrm{B})$ pathways (23). A number of transcriptional factors are activated through these pathways, and eventually, the cells undergo metabolic adaptation, resulting in increased cell proliferation, survival and differentiation into plasma or memory B cells, as well as antibody production $(24,25)$. Most recently, inhibitors of BCR signaling have become an area of substantial clinical interest, particularly in chronic lymphocytic leukemia (CLL), which is likely attributed to its role in BCR- and chemokine-controlled integrin-mediated adhesion and the homing of malignant $\mathrm{B}$ cells to lymph node and bone marrow microenvironments that support growth and survival $(26,27)$. Collectively, these data support the fact that BCR activation not only controls intrinsic survival pathways associated with B lymphoma cells, but that it also regulates stroma-mediated extrinsic lymphoma cell survival, and lymphoma cell homing and interplay with the microenvironment. Targeting the BCR signal pathway molecules will block the growth and survival signals emanating from cell intrinsic mutations and the microenvironment, and is therefore a promising therapeutic strategy for lymphoma therapy.

BTK is a member of the tyrosine-protein (Tec) family of kinases, expressed in hematopoietic cells, particularly in B cells, but not in T cells or normal plasma cells (28). BTK is activated by the upstream Src-family and leads to the downstream activation of essential cell survival pathways, such as NF- $\mathrm{BB}$ and MAPK. Furthermore, it plays an important role in the signaling pathways of $\mathrm{C}-\mathrm{X}-\mathrm{C}$ motif chemokine ligand 12-chemokine (C-X-C motif) receptor 4; (CXCL12-CXCR4), B-cell activating factor (BAFF) receptor, $\mathrm{Fc} \gamma$ receptor $(\mathrm{Fc} \gamma \mathrm{R})$, Toll-like receptor and receptor activator of NF- $\mathrm{KB}$, which prompt B-cell migration, adhesion, self-tolerance, inmmune activation and cytokine secretion (29-32). Deficient BTK is associated with its loss of function and reduced mature B cell numbers, and results in X-linked agammaglobulinemia. Ibrutinib, an oral irreversible BTK inhibitor, is designed to bond to a Cys481 residue within the BTK active site, preventing the Tyr223 phosphorylation required for its activation (33). The drug was approved by the Food and Drug Administration in February 2013 for several clinical trials on various forms of B-cell malignancies. Notably, marked efficacy for ibrutinib has been noted thus far, particularly in CLL/small lymphocytic lymphoma (SLL) and mantel cell lymphoma (MCL).

\section{Preclinical data on ibrutinib in the treatment of B-cell malignancies}

In vitro experiments revealed that ibrutinib significantly inhibited CLL cell survival, DNA synthesis and migration in response to tissue homing chemokines (CXCL12 and CXCL13), and that it effectively blocked survival signals, which are provided externally to CLL cells from the microenvironment [CD40L, BAFF, interleukin (IL)-6, IL-4 and tumor necrosis factor- $\alpha$ ] $(34,35)$. Ibrutinib also downregulated the BCR-dependent chemokines [chemokine (C-C motif) ligand 3 (CCL3) and CCL4] by CLL cell secretion $(36,37)$. Ibrutinib strongly inhibited the survival of malignant cells, including 
CLL, MCL, diffuse large B-cell lymphoma (DLBCL), follicular lymphoma (FL) and multiple myeloma (MM) cells, in in vivo and in vitro experiments (38-40). In addition, ibrutinib and bortezomib synergistically kill activated B cell-like-DLBCL (ABC-DLBCL) or germinal-center B-cell-like DLBCL (GC-DLBCL) cells and MCL cells, including those highly resistant to bortezomib, but not normal cells (41). Furthermore, Rushworth et al (42) reported for the first time that ibrutinib treatment significantly augments the cytotoxic activity of bortezomib and lenalidomide chemotherapies by inhibiting the NF- $\mathrm{KB}$ pathway in malignant plasma cells from patients with MM, which may provide a theoretical basis for future combination therapy. Notably, ibrutinib was also shown to disrupt the chemokine-induced adhesion and migration of primary chronic leukemia B cells (43).

In a mouse xenograft in vivo model, ibrutinib inhibited CLL progression. As in humans, the mice exhibited transient lymphocytosis at day 4 and a reduction in tumor size, demonstrating that CLL cells can be mobilized into peripheral blood from lymphoid tissue by ibrutinib (33). This is likely as the protection from the TME is prevented and indicates that ibrutinib may overcome the drug resistance mediated by the microenvironment. As canine non-Hodgkin's lymphoma (NHL) shares a number of characteristics with human NHL, including diagnostic classifications and response to cyclophosphamide, doxorubicin, vincristine and prednisone/prednisolone regimen-based chemotherapy (44), Honigberg et al (45) treated treatment-naïve (TN) and relapsed dogs with ibrutinib using the capsule formulation prepared for human clinical trials. The study showed that ibrutinib led to objective clinical responses [3 partial response (PR) and 3 stable disease (SD)] in spontaneous canine B-cell lymphomas by response evaluation criteria. Total Btk levels varied significantly across samples, which may suggest heterogeneity in biopsy sampling in tumor cells.

Taken together, these $e x$ vivo and in vivo studies demonstrated that BCR/BTK is a central mediator of malignant B-cell homing, survival and microenvironment-mediated drug resistance. Further support for this conclusion may be observed in the marked responses of B-cell disorders treated with ibrutinib alone or in combination with cytotoxic agents.

\section{Clinical trials of ibrutinib in the treatment of B-cell ma- lignancies using monotherapy and combination regimens}

In a multi-cohort phase Ib/II trial of ibrutinib in $\mathrm{TN}$ or relapsed/refractory (RR) CLL/SLL patients, $83 \%$ of patients with RR disease and $96 \%$ of treatment-naïve patients, including those with high-risk disease, were estimated to experience overall survival (OS) times of 26 months (46). The study demonstrated that ibrutinib may be the first-line treatment for previously untreated CLL patients. Notably, the high- and low-dose groups achieved the same efficacy in this trial, which suggested that the efficacy was not positively correlated with the dose of ibrutinib but may be associated with the complete suppression of BTK. Advani et al (47) confirmed this hypothesis and presented a study in which patients with RR B-cell lymphoma and CLL received escalating oral doses of ibrutinib (1.25-12.5 mg/kg per day). BTK occupancy $>95 \%$ was observed in dose level cohorts II to V (2.5-12.5 mg/kg per day), and each of these cohorts experienced similar response rates, consistent with the efficacy derived from BTK inhibition. Notably, during the first treatment cycle, all CLL patients experienced rapid reductions in lymphadenopathy accompanied by an increase in absolute lymphocyte count, indicating that the malignant cells were moving from the lymph nodes into the peripheral blood. The results suggested that old response criteria for progressive disease based on lymphocytosis may have to be modified, since lymphocytosis associated with inhibitors targeting the BCR pathway is clearly not a sign of disease progression. To this end, National Comprehensive Cancer Network guidelines have eliminated progressive lymphocytosis as a sign of disease progression when spleen and lymph node sizes are reduced (48).

Following this, to confirm the efficacy of ibrutinib in patients with RR MCL, Wang et al (49) conducted a phase II study, with 109 RR MCL patients (63 bortezomib-naïve and 46 bortezomib-exposed). The overall response rate (ORR) is $68 \%$ according to the International Working Group response criteria (49), and in the study by Wang et al, the ORR was $65 \%$ in the bortezomib-naive cohort and $72 \%$ in the bortezomib-exposed cohort. The data from this phase II trial showed that the single agent ibrutinib is highly active in R/R MCL. Moreover, patients who received ibrutinib therapy following bortezomib treatment experienced improved treatment efficacy, suggesting that combining the drug with other therapies deserves further study, which may be of great patient benefit. Staudt et al (50) reported a study in which 8 patients with RR ABC DLBCL were enrolled, with a complete response (CR) recorded in 2 patients (25\%), SD in 3 patients (37\%) and progressive disease in 3 patients (38\%). Notably, 1 patient with primary refractory disease achieved SD with ibrutinib, associated with a $25 \%$ tumor reduction, and is currently in $\mathrm{CR}$ following allogeneic bone marrow transplantation. CD79B mutations, which cause chronic BCR signaling in ABC DLBCL, were revealed in 2 patients; 1 patient with SD who achieved a $25 \%$ tumor response and another who achieved a CR. However, ibrutinib was shown to significantly improve survival only in ABC DLBCL but not GC DLBCL patients. Another phase 2 study also indicated that ibrutinib showed preferential response activity in $\mathrm{ABC}$ versus $\mathrm{GCB}$ DLBCL (Table I) (51). Thus, chronic active BCR signaling maybe a significant therapeutic target in ABC DLBCL or other B-cell malignancies. In FL, when ibrutinib was administered orally with dose escalation in 16 patients with FL (52), the ORR was $54.5 \%$ ( $3 \mathrm{CR}$ and $3 \mathrm{PR}$ ), the duration of response was 12.3 months and the median progression-free survival time was 13.4 months, showing promising treatment efficacy for this disease. These clinical experiments showed that the side effects of ibrutinib are well tolerated. Grade 1 or 2 diarrhea, fatigue and nausea have been the most frequently reported adverse events (AEs). Grade 3 AEs included anemia, anxiety, hypersensitivity, hypokalemia, hypophosphatemia and decreased neutrophil count, while grade 4 hypokalemia was also considered to be associated with ibrutinib $(53,54)$. As more experience is gained with this agent, the patients who will benefit the most will be chosen. Further research is required not only to identify the response biomarkers and the mechanism of resistance, but also to understand how these agents may be rationally combined. 
Table I. Basic characteristics of lymphoma and efficacy of ibrutinib.

\begin{tabular}{|c|c|c|c|c|c|c|}
\hline Diseases & Patients, $\mathrm{n}$ & Age, years & Doses & $\mathrm{AE}$ & ORR & Study phase \\
\hline CLL & 61 & $\geq 65$ & $\begin{array}{l}420 \text { or } \\
840 \mathrm{mg}\end{array}$ & $\begin{array}{l}\text { Diarrhea, fatigue, } \\
\text { rash; serious AEs } \\
\text { occurred in } 10 \% \\
\text { of patients }\end{array}$ & $\begin{array}{c}70 \% \\
\text { (420-mg cohort) } \\
63 \% \\
\text { (840-mg cohort) }\end{array}$ & $\mathrm{Ib} / \mathrm{II}$ \\
\hline MCL & 109 & $68(40-84)^{\mathrm{a}}$ & $\begin{array}{l}560 \mathrm{mg} \\
(\mathrm{po} \mathrm{qd})\end{array}$ & $\begin{array}{l}\text { Diarrhea, fatigue, } \\
\text { upper respiratory } \\
\text { tract infection, } \\
\text { dyspneaoedema } \\
\text { peripheral, grade } 5 \\
\text { pneumonia }\end{array}$ & $\begin{array}{c}65 \% \\
\text { (bortezomib-naïve) } \\
67 \% \\
\text { (bortezomib-exposed) }\end{array}$ & II \\
\hline DLBCL & 70 & $60(41-71)^{\mathrm{a}}$ & $\begin{array}{l}560 \mathrm{mg} \\
(\text { po qd) }\end{array}$ & $\begin{array}{l}\text { AEs consistent } \\
\text { with that reported } \\
\text { in other ibrutinib } \\
\text { studies }\end{array}$ & $\begin{array}{c}40 \% \\
\text { (ABC subtype) } \\
5 \% \\
\text { (GCB subype) }\end{array}$ & II \\
\hline FL & 16 & $60(41-71)^{\mathrm{a}}$ & $\begin{array}{c}1.25-12.5 \\
\mathrm{mg} / \mathrm{kg}\end{array}$ & $\begin{array}{l}\text { Diarrhea, fatigue } \\
\text { nausea, coughing }\end{array}$ & $54.5 \%$ & I \\
\hline
\end{tabular}

Patients with relapsed or refractory MCL who were either bortezomib-naïve or bortezomib-exposed (prior treatment with at least 2 cycles of bortezomib) were eligible for study. PCI-32765 (ibrutinib) was administered orally at $560 \mathrm{mg}$ daily (in continuous 28-day cycles) until disease progression halted. The tumor response was evaluated every 2 cycles and classified by the 2007 non-Hodgkin's lymphoma International

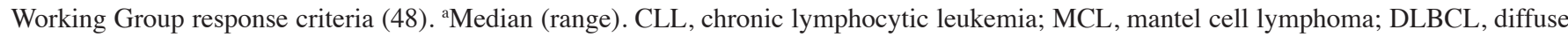
large B-cell lymphoma; FL, follicular lymphoma; po, by mouth; qd, every day; AE, adverse event; ORR, overall response rate; ABC, activated $\mathrm{B}$ cell; GCB, germinal-center B-cell.

Table II. Comparison of the efficacy of ibrutinib in monotherapy and combination therapy.

\begin{tabular}{lrrrr}
\hline Study & $\mathrm{n}$ & ORR, $\%$ & CR, \% & PFS \\
\hline $\begin{array}{l}\text { Single agent } \\
\text { RR }\end{array}$ & 61 & 67 & 4 & $88 \%$ at 18 months \\
TN & 31 & 74 & 10 & $96 \%$ at 15 months \\
Combination & & & & \\
PCI+BR & 30 & 93 & 13 & $90 \%$ at 11 months \\
PCI+FCR & 3 & 100 & 67 & $100 \%$ at 11 months $^{\text {a }}$ \\
PCI+ofatumumab & 27 & 100 & 4 & $89 \%$ at 11 months $^{\text {a }}$ \\
\hline
\end{tabular}

ORR, overall response rate; $\mathrm{CR}$, complete response; PFS, progression-free survival; RR, relapsed/refractory; TN, treatment-naïve; PCI, ibrutinib; BR, bendamustine and rituximab regimen; FCR, fludarabine/Cytoxan/Rituxan regimen. ${ }^{\text {aThe clinical trial }}$ (NCT01217749) (57).

The most comprehensive study of ibrutinib has been performed in CLL. Results from a phase 1/2 trial suggested that high- and low-risk CLL patients respond equally as well to ibrutinib (55). CLL patients treated with single-agent ibrutinib therapy characteristically exhibit delayed responses or SD. To accelerate and improve these responses, a phase 2 single-center clinical trial of ibrutinib plus rituximab was conducted, which accrued 40 patients. In total, 32 patients with unmutated immunoglobulin heavy chain variable, 20 patients with del17p or tumor protein p53 mutation (4 without prior therapy) and 13 patients with del11q were enrolled. From the 20 patients in whom an early response assessment could be evaluated at 3 months, 17 patients achieved a partial remission for an ORR of $85 \%$, and 3 achieved a PR. However, in this combination trial, it was noted that the re-distribution lymphocytosis peaked earlier and with a shorter duration compared with single-agent ibrutinib (42), which was possibly due to the addition of rituximab. Treatment was well tolerated, with diarrhea, bone pain and fatigue as the most frequent side effects. In addition, another study (56) showed that ibrutinib in combination with ofatumumab is well-tolerated and highly active in patients with RR CLL (ORR, 100\%) irrespective of prognostic markers (Table II). A further study (57) was performed to test ibrutinib in combination with a bendamustine and rituximab regimen (BR) or a fludarabine/Cytoxan/Rituxan regimen (FCR) in patients with RR CLL. The results indicated that at a median follow-up time of 4.9 months, 16 patients had completed BR therapy and 14 patients were still receiving BR therapy, with an ORR of $93 \%$ (28/30 patients; CR, $13 \%$ and PR $80 \%$ ). The responses appeared to be independent of high-risk clinical or genomic features. The majority of patients $(77 \%)$ remained a part of the study at the time the data was presented. While only 3 patients were included in the FCR+ibrutinib cohort, the therapy was well tolerated, with only one serious AE, which was neutropenic fever. The ORR was $100 \%$ (3/3) and the 2 confirmed minimal residual disease (MRD)-negative cases achieved CRs (57).

Although the number of patients receiving combination therapy and the number of associated clinical experiments is small, the benefits that CLL patients have gained is clear. Next, 
the optimal combination strategies must be chosen according to the accurate molecular classification of patients or other criteria, such as disease progression, leading to personalized therapy. The clinical data on combination therapy using ibrutinib in patients with MCL, DLBCL, MM and FL is limited; however, these pre-clinical experiments on CLL provide the rationale for using a combination strategy for other types of B cell malignancies.

\section{Conclusions}

Over the last three years, the progress in the study of pathological BCR signaling in lymphoma has resulted in the rapid development of BCR pathway inhibitors. The compounds that have been developed furthest in the clinic are the inhibitors of SYK, mTOR, PI3K- $\delta$ and BTK. To date, marked success has been achieved by these therapeutic agents in the treatment of patients with B cell malignancies who, in a number of cases, were resistant to conventional chemotherapeutic agents. Furthermore, the side effect profile of BCR-targeted therapies appears to be easily manageable. However, despite high ORRs for BCR pathway inhibitors, a substantial minority of patients is unresponsive or shows progression relatively soon after the commencement of therapy. Therefore, the identification of molecular mechanisms that may predict and sustain the response to BCR pathway inhibitors would be beneficial in order to overcome drug resistance. The BCR inhibitors, as discussed for the aforementioned BTK inhibitor, target the extracellular and intracellular determinants of the bone marrow niche. The aim of associated studies is to identify the appropriate factors to target within the complex network of the tumor cell microenvironment. To this end, therapies that are able to overcome the coordinated effort between lymphoma cells and the microenvironment are required. In this way, the sequence of events (de novo and acquired) facilitating MRD and ending in therapy resistance may be interrupted. Going forward, it is important to note that with the significant heterogeneity of signaling factors and transduction pathways within the TME (bone marrow and lymph node) niche, the designing of combination therapies with targeted agents is required. To this end, the targeting of multiple pathways, either simultaneously or in sequence, may be the only measure by which to overcome the sanctuary of the TME milieu, since these pathways act in concert in lymphomagenesis. It will be critical to merit the combined targeting of downstream (BCR) signaling pathways to maintain the advantage of direct modulation of the cell survival and proliferation machinery, with targeting of upstream or parallel pathways to circumvent the compensatory survival pathway. As a result, the success of BCR inhibitor therapy in B-cell lymphoma therapy will be dependent on the use of rational combinations of targeted agents, and good knowledge of the nature of signaling pathways and their interactions with the TME.

\section{Acknowledgements}

The present study was supported by grants from the National Natural Science Foundation of China (nos. 30672208 and 81270603).

\section{References}

1. Younes A: Beyond chemotherapy: New agents for targeted treatment of lymphoma. Nat Rev Clin Oncol 8: 85-96, 2011.

2. Manzur S, Cohen S, Haimovich J and Hollander N: Enhanced therapeutic effect of B cell-depleting anti-CD20 antibodies upon combination with in-situ dendritic cell vaccination in advanced lymphoma. Clin Exp Immunol 170: 291-299, 2012.

3. Friedberg JW: New strategies in diffuse large B-cell lymphoma: Translating findings from gene expression analyses into clinical practice. Clin Cancer Res 17: 6112-6117, 2011.

4. Timmerman JM, Byrd JC, Andorsky DJ, et al: A phase I dose-finding trial of recombinant interleukin-21 and rituximab in relapsed and refractory low grade B-cell lymphoproliferative disorders. Clin Cancer Res 18: 5752-5760, 2012.

5. Fowler N and Oki Y: Developing novel strategies to target B-cell malignancies. Am Soc Clin Oncol Educ Book, 366-372, 2013.

6. Ramsay AD and Rodriguez-Justo M: Chronic lymphocytic leukaemia-the role of the microenvironment pathogenesis and therapy. Br J Haematol 162: 15-24, 2013.

7. Gupta P, Goldenberg DM, Rossi EA and Chang CH: Multiple signaling pathways induced by hexavalent, monospecific, anti-CD20 and hexavalent, bispecific, anti-CD20/CD22 humanized antibodies correlate with enhanced toxicity to B-cell lymphomas and leukemias. Blood 116: 3258-3267, 2010.

8. Roschewski M, Dunleavy K and Wilson WH: Diffuse large B cell lymphoma: Molecular targeted therapy. Int J Hematol 96: 552-561, 2012.

9. Kenkre VP and Kahl BS: The future of B-cell lymphoma therapy: The B-cell receptor and its downstream pathways. Curr Hematol Malig Rep 7: 216-220, 2012.

10. Sison EA, Rau RE, McIntyre E, Li L, Small D and Brown P: MLL-rearranged acute lymphoblastic leukaemia stem cell interactions with bone marrow stroma promote survival and therapeutic resistance that can be overcome with CXCR4 antagonism. Br J Haematol 160: 785-797, 2013.

11. Chen L, Monti S, Juszczynski P, et al: SYK inhibition modulates distinct PI3K/AKT-dependent survival pathways and cholesterol biosynthesis in diffuse large B cell lymphomas. Cancer Cell 23: 826-838, 2013.

12. Irish JM, Myklebust JH, Alizadeh AA, et al: B-cell signaling networks reveal a negative prognostic human lymphoma cell subset that emerges during tumor progression. Proc Natl Acad Sci USA 107: 12747-12754, 2010.

13. Young RM and Staudt LM: Targeting pathological B cell receptor signalling in lymphoid malignancies. Nat Rev Drug Discov 12: 229-243, 2013.

14. Shain KH and Tao J: The B-cell receptor orchestrates environment-mediated lymphoma survival and drug resistance in B-cell malignancies. Oncogene 33: 4107-4113, 2014.

15. Rickert RC: New insights into pre-BCR and BCR signalling with relevance to B cell malignancies. Nat Rev Immunol 13: 578-591, 2013.

16. Choi MY and Kipps TJ: Inhibitors of B-cell receptor signaling for patients with B-cell malignancies. Cancer J 18: 404-410, 2012.

17. Stevenson FK, Krysov S, Davies AJ, et al: B-cell receptor signaling in chronic lymphocytic leukemia. Blood 118: 4313-4320, 2011.

18. Chen L, Huynh L, Apgar J, et al: ZAP-70 enhances IgM signaling independent of its kinase activity in chronic lymphocytic leukemia. Blood 111: 2685-2692, 2008.

19. Depoil D, Fleire S, Treanor BL, et al: CD19 is essential for $\mathrm{B}$ cell activation by promoting $\mathrm{B}$ cell receptor-antigen microcluster formation in response to membrane-bound ligand. Nat Immunol 9: 63-72, 2008.

20. Niiro $H$ and Clark EA: Regulation of B-cell fate by antigen-receptor signals. Nat Rev Immunol 2: 945-956, 2002.

21. Humphries LA, Dangelmaier C, Sommer K, et al: Tec kinases mediate sustained calcium influx via site-specific tyrosine phosphorylation of the phospholipase Cgamma Src homology 2-Src homology 3 linker. J Biol Chem 279: 37651-37661, 2004.

22. Hashimoto A, Okada H, Jiang A, et al: Involvement of guanosine triphosphatases and phospholipase C-gamma2 in extracellular signal-regulated kinase, c-Jun NH2-terminal kinase, and p38 mitogen-activated protein kinase activation by the $\mathrm{B}$ cell antigen receptor. J Exp Med 188: 1287-1295, 1998.

23. Shinohara M, Koga T, Okamoto K, et al: Tyrosine kinases Btk and Tec regulate osteoclast differentiation by linking RANK and ITAM signals. Cell 132: 794-806, 2008. 
24. Davis RE, Ngo VN, Lenz G, et al: Chronic active B-cell-receptor signalling in diffuse large B-cell lymphoma. Nature 463: 88-92, 2010.

25. Khan WN: Regulation of B lymphocyte development and activation by Bruton's tyrosine kinase. Immunol Res 23: 147-156, 2001

26. Hamblin TJ, Davis Z, Gardiner A, Oscier DG and Stevenson FK: Unmutated $\operatorname{Ig} \mathrm{V}(\mathrm{H})$ genes are associated with a more aggressive form of chronic lymphocytic leukemia. Blood 94: 1848-1854, 1999.

27. Gobessi S, Laurenti L, Longo PG, et al: ZAP-70 enhances B-cell-receptor signaling despite absent or inefficient tyrosine kinase activation in chronic lymphocytic leukemia and lymphoma B cells. Blood 109: 2032-2039, 2007.

28. Genevier HC, Hinshelwood S, Gaspar HB, et al: Expression of Bruton's tyrosine kinase protein within the B cell lineage. Eur J Immunol 24: 3100-3105, 1994.

29. Vargas L, Hamasy A, Nore BF and Smith CI: Inhibitors of BTK and ITK: State of the new drugs for cancer, autoimmunity and inflammatory diseases. Scand J Immunol 78: 130-139, 2013.

30. O'Hayre M, Salanga CL, Kipps TJ, et al: Elucidating the CXCL12/CXCR4 signaling network in chronic lymphocytic leukemia through phosphoproteomics analysis. PLoS One 5: e11716, 2010.

31. Redondo-Muñoz J, Escobar-Díaz E, Samaniego R, et al MMP-9 in B-cell chronic lymphocytic leukemia is up-regulated by alpha4beta1 integrin or CXCR4 engagement via distinct signaling pathways, localizes to podosomes, and is involved in cell invasion and migration. Blood 108: 3143-3151, 2006

32. Richards KL, Motsinger-Reif AA, Chen HW, et al: Gene profiling of canine B-cell lymphoma reveals germinal center and postgerminal center subtypes with different survival times, modeling human DLBCL. Cancer Res 73: 5029-5039, 2013.

33. Herman SE, Sun X, McAuley EM, et al: Modeling tumor-host interactions of chronic lymphocytic leukemia in xenografted mice to study tumor biology and evaluate targeted therapy. Leukemia 27: 2311-2321, 2013.

34. Chang BY, Huang MM, Francesco M, et al: The Bruton tyrosine kinase inhibitor PCI-32765 ameliorates autoimmune arthritis by inhibition of multiple effector cells. Arthritis Res Ther 13: R115, 2011

35. Lee KG, Xu S, Wong ET, Tergaonkar V and Lam KP: Bruton's tyrosine kinase separately regulates NFkappaB p65RelA activation and cytokine interleukin (IL)-10/IL-12 production in TLR9-stimulated B cells. J Biol Chem 283: 11189-11198, 2008.

36. Mráz M, Doubek M and Mayer J: Inhibition of B cell receptor signaling: A first targeted therapeutic approach for chronic lymphocytic leukemia and other B cell lymphomas. Klin Onkol 26: 179-185, 2013 (In Czech).

37. Tai YT, Chang BY, Kong SY, et al: Bruton tyrosine kinase inhibition is a novel therapeutic strategy targeting tumor in the bone marrow microenvironment in multiple myeloma. Blood 120: $1877-1887,2012$

38. Herman SE, Gordon AL, Hertlein E, et al: Bruton tyrosine kinase represents a promising therapeutic target for treatment of chronic lymphocytic leukemia and is effectively targeted by PCI-32765. Blood 117: 6287-6296, 2011.

39. Schwamb J, Feldhaus V, Baumann M, et al: B-cell receptor triggers drug sensitivity of primary CLL cells by controlling glucosylation of ceramides. Blood 120: 3978-3985, 2012.

40. Yang Y, Shaffer AL III, Emre NC, et al: Exploiting synthetic lethality for the therapy of $\mathrm{ABC}$ diffuse large $\mathrm{B}$ cell lymphoma. Cancer Cell 21: 723-737, 2012

41. Dasmahapatra G, Patel H, Dent P, et al: The Bruton tyrosine kinase (BTK) inhibitor PCI-32765 synergistically increases proteasome inhibitor activity in diffuse large-B cell lymphoma (DLBCL) and mantle cell lymphoma (MCL) cells sensitive or resistant to bortezomib. Br J Haematol 161: 43-56, 2013.

42. Rushworth SA, Bowles KM, Barrera LN, et al: BTK inhibitor ibrutinib is cytotoxic to myeloma and potently enhances bortezomib and lenalidomide activities through NF- $\kappa$ B. Cell Signal 25: 106-112, 2013.
43. de Rooij MF, Kuil A, Geest CR, et al: The clinically active BTK inhibitor PCI-32765 targets B-cell receptor- and chemokine-controlled adhesion and migration in chronic lymphocytic leukemia. Blood 119: 2590-2594, 2012.

44. Sabine P, Balasubramanian S, Pham LV, et al: Activity of Bruton's tyrosine kinase (Btk) inhibitor PCI-32765 in mantle cell lymphoma (MCL) identifies Btk as a novel therapeutic target. Blood (ASH Annual Meeting Abstracts) 118: 3688, 2011.

45. Honigberg LA, Smith AM, Sirisawad M, et al: The Bruton tyrosine kinase inhibitor PCI-32765 blocks B-cell activation and is efficacious in models of autoimmune disease and B-cell malignancy. Proc Natl Acad Sci USA 107: 13075-13080, 2010.

46. O'Brien S, MD, Furman RR, Coutre SE, et al: Ibrutinib as initial therapy for elderly patients with chronic lymphocytic leukaemia or small lymphocytic lymphoma: An open-label, multicentre, phase 1b/2 trial. Lancet Oncol 15: 48-58, 2014.

47. Advani RH, Buggy JJ, Sharman JP, et al: Bruton tyrosine kinase inhibitor ibrutinib (PCI-32765) has significant activity in patients with relapsed/refractory B-cell malignancies. J Clin Oncol 31: 88-94, 2013.

48. Byrd JC, Furman RR, Coutre SE, et al: Targeting BTK with Ibrutinib in Relapsed Chronic Lymphocytic Leukemia. N Engl J Med 369: 32-42, 2013

49. Wang L, Martin P, Blum KA, et al: The Bruton's tyrosine kinase inhibitor PCI-32765 is highly active as single-agent therapy in previously-treated mantle cell lymphoma (MCL): Preliminary results of a phase II trial. Blood (ASH Annual Meeting Abstracts) 118: 442, 2011.

50. Staudt LM, Dunleavy K, Buggy JJ, et al: The Bruton's tyrosine kinase (BTK) inhibitor PCI-32765 modulates chronic active BCR signaling and induces tumor regression in relapsed/refractory ABC DLBCL. Blood (ASH Annual Meeting Abstracts) 118: 3174, 2011.

51. Wilson WH, Gerecitano JF, Goy A, et al: The Bruton's tyrosine kinase (BTK) inhibitor, Ibrutinib (PCI-32765), Has Preferential activity in the $\mathrm{ABC}$ subtype of relapsed/refractory de novo diffuse large B-cell lymphoma (DLBCL): Interim results of a multicenter, open-label, phase 2 study. Blood (ASH Annual Meeting Abstracts) 120: 686, 2012.

52. Fowler NH, Advani RH, Sharman JP, et al: The Bruton's tyrosine kinase inhibitor ibrutinib (PCI-32765) is active and tolerated in relapsed follicular lymphoma. Blood (ASH Annual Meeting Abstracts) 120: 156, 2012

53. Jaglowski SM, Jones JA, Flynn JM, et al: A phase Ib/II study evaluating activity and tolerability of BTK inhibitor PCI-32765 and ofatumumab in patients with chronic lymphocytic leukemia/small lymphocytic lymphoma (CLL/SLL) and related diseases. J Clin Oncol (2012 ASCO Annual Meeting) 30: 6508, 2012.

54. O'Brien S, Burger JA, Blum KA, et al: The Bruton's tyrosine kinase (BTK) inhibitor PCI-32765 induces durable responses in relapsed or refractory $(\mathrm{R} / \mathrm{R})$ chronic lymphocy tic leukemia/small lymphocytic lymphoma (CLL/SLL): Follow-up of a phase Ib/II study. Blood (ASH Annual Meeting Abstracts) 120: 983, 2011.

55. Burger JA, Keating MJ, Wierda WG, et al: Safety and activity of ibrutinib plus rituximab for patients with high-risk chronic lymphocytic leukaemia: A single-arm, phase 2 study. Lancet 15: 1090-1099, 2014.

56. O'Brien SM, Barrientos JC, Flinn IW, et al: Combination of the Bruton's tyrosine kinase (BTK) inhibitor PCI-32765 with bendamustine(B)/rituximab (R) (BR) in patients (pts) with relapsed/refractory (R/R) chronic lymphocytic leukemia (CLL): Interim results of a phase Ib/IIstudy. J Clin Oncol (2012 ASCO Annual Meeting) 30: 6515, 2012.

57. Brown JR, BJ, Flinn I, et al: The Bruton's tyrosine kinase (BTK) inhibitor ibrutinib combined with bendamustine and rituximab is active and tolerable in patients with relapsed/refractory CLL, interim results of a phase Ib/II study. EHA Meeting Abstracts 97: 2012. 